Ethiopian Journal of Environmental Studies \& Management 10(3): 361 - 377, 2017.

ISSN:1998-0507

doi: https://dx.doi.org/10.4314/ejesm.v10i3.7

Submitted: December 25, 2016

Accepted: April 27, 2017

\title{
DIVERSITY OF BENTHIC BIOTA OF SOME FRESHWATER HABITATS IN KABBA/BUNU LOCAL GOVERNMENT, KOGI STATE, NORTH-CENTRAL, NIGERIA
}

\author{
*ADESALU, T.A., ${ }^{1}$ KUNRUNMI, O.A. ${ }^{1}$ AND LAWAL, M.0. ${ }^{2}$ \\ ${ }^{1}$ Department of Botany, University of Lagos, Nigeria \\ ${ }^{2}$ Department of Marine Sciences, University of Lagos, Nigeria
}

\begin{abstract}
The composition and diversity of different water bodies in Kaaba/Bunu Local Government, Kogi State were investigated for both micro and macrobiota. The study covered Oinyi, Odogo and Odonkolo Rivers. Salinity values ranged between $0.03 \%$ and $0.07 \%$ indicating the freshwater status of these water bodies, $\mathrm{pH}$ was between 6.50 and 7.61. Conductivity values were between $88.1 \mu \mathrm{S} / \mathrm{cm}$ and $131.7 \mu \mathrm{S} / \mathrm{cm}$ while dissolved oxygen highest value $(5.12 \mathrm{mg} / \mathrm{L})$ was recorded at Odonkolo River. For the phytobenthos, eighty-five taxa recorded were distributed among five divisions, Bacillariophyta, Chlorophyta, Charophyta, Euglenophyta and Cyanobacteria. Bacillariophyta (Diatoms) accounted for $78.28 \%$ of the identified species, followed by the Charophytes, (6.99\%), Chlorophytes (5.61\%), Euglenophytes (7.43\%) and Cyanobacteria (1.69\%). Macrobenthic invertebrates recorded three group; gastropoda (37.71\%), insecta (28.57\%) and oligochaeta (35.71\%).
\end{abstract}

Key Words: Phytobenthos, macrobenthic, Water, River, Diversity, Kogi State

\section{Introduction}

Water bodies like rivers, are important by being involved in maintaining a balance in the ecosystem by supporting diverse plankton and other organisms in the food chain. Algae are important primary producers, since phytoplankton communities produce approximately half of the global net primary production (Field et al., 1998) as cited by Marinković et al. (2016). According to the European Water framework Directive (WFD 2000), phytoplankton and phytobenthos are used in the determination of water quality. They help to improve water quality and serve as nursery grounds for fish and crabs. Benthic algae, according to Makovinsca and Hlubikova (2015) are the most successful primary producers in aquatic habitats. They are important chemical modulators, transforming inorganic chemicals into organic forms, (Molholland, 1996) functions as stabilizers of substrata and serve as important habitat for many organisms (Makovinsca and Hlubikova, 2015). Biggs et al. (1998) were of the opinion that benthic algal community structure is driven by light and nutrients availability and disturbances which is hydrological stress. According to George et al. (2009)

*Corresponding Author: Adesalu, T.A.

Email: tadesalu@unilag.edu.ng 
cited by Esenowo and Ugwumba (2010), macrobenthos play an important role in aquatic community which includes mineralization, mixing of sediments and flux of oxygen into sediment, cycling of organic matter and assessing the quality of inland water. The ecological problems in Kogi State include leaching, erosion and general impoverishment of the soil. These problems are compounded by the annual bush burning of the savanna that further exposed the top soil to more erosion. (Adeoye, 2012). This study was undertaken in order to have the baseline biota of these rivers since no phycological work has been done in these waters.

\section{Materials and Methods \\ Description of Study Sites}

The study site is located at the northern part of Kabba town, Alape road along Kabba-Ilorin road, Kabba, Kogi state (Fig 1.), Nigeria. Kogi state is characterized as a sub humid zone with derived savannah vegetation, patches of rainforest and harsh tolerance plant species alongside with economic trees and agricultural crops. Kabba/Bunu Local government part of the study site is described as Southern Guinea Savanna zone of secondary forest with dominant type being savanna woodland consisting of trees of varying species and scanty grass cover with a number of small sized farms growing a mix of cassava, yams, maize and cowpeas. Riparian vegetation along the study sites consist of Raphia hookeri G. Mann and H.Wendl, Pterocarpus erinaceus Poir, Anchomanes difformis (Blume) Engl, Anogeissus leiocarpus (DC.) Guill. And Perr., Detarium macrocarpum Harms, Eriosema psoralioides (Lam) G. Don,
Sporobolus pyramidalis P. Beauv.,Tephrosia linearis (Willd.) Pers., Urena lobata L., Vitex doniana Sw., Holarrhena floribunda (G.Don.) Dur. \& Schinz, Mimosa pudica L., Margaritaria discoidea (Baill.) Webster, Hyptissua veolens (L.) Poit, Byrsocarpus coccineus Schum. And Thonn., Canavalia ensiformis (L.) DC.), Waltheria indica L. The rivers are seasonal and partly dry up during the dry season but are very deep and wide during the wet season. These rivers serve as the main source of water for the inhabitants of the environment especially during wet season. During the wet season, the inhabitants carry out fishing activities in the river. Farming and Lumbering is the major activity at the study sites.

Oinyi River: Is a protected area, is in Oinyi Forestry Reserve and after Okpa area. The River is said to reach a depth of about $34 \mathrm{~m}$ if traced further down. However, this particular location was more of a stream measuring $0.23 \mathrm{~m}$ depth. The stream was covered by high forest vegetation (Plate 1 (A and $B)$ ). It was difficult to navigate further down because of the roughness of the terrain. The vegetation around this water body is dominated by Tectona grandis, Gmelina arborea and Mimosa pudica was seen growing abundantly at this site. Seven stations with GPS readings of $\mathrm{N}^{\circ} 8^{\circ}$ 00.322' E006 ${ }^{\circ}$ 17.231' (Station 1); N070 58.005' E006 $15.811^{\prime}$ (Station 2); N08 00. 213' E006 ${ }^{\circ} 16.989^{\prime}$ (Station3); N08 00. 253' E006 $17.419^{\prime}$ (Station 4); N08 00. 262' E006 16.911' (Station 5); N08 00. 538' E006'16.690' (Station 6) and N070 57. 296' E006 ${ }^{\circ}$ 14. 419' (Station 7) were created. The sediment is sandy. 
Other Rivers: Some of the location shows evidence of erosion. The water was cloudy as a result of leaching of clay soil into the stream. The sediment was sandy with lot of stones for most of the water bodies around the study area. The vegetation around this area is similar to that describe above. Most rivers at the study site especially Odogu and Orioyo water are gravel bed river (Plate 1 ( $\mathrm{C}$ and D)). Odonkolo River bank is densely populated with high forest plant species, the water looks clear; the depth and transparency measurements were the same $(0.27 \mathrm{~m})$. Three stations with GPS

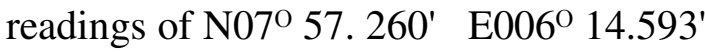
(Station 8); (N07O 57. 454' E006 ${ }^{\circ}$ 15.250' (Station9); N070 56. 578' E006 ${ }^{\circ}$ 14. 966' (Station 10) for Odogu and Orioyo River while Odonkolo has one station N070 58. 956' E006 20. 769' (Station 11).

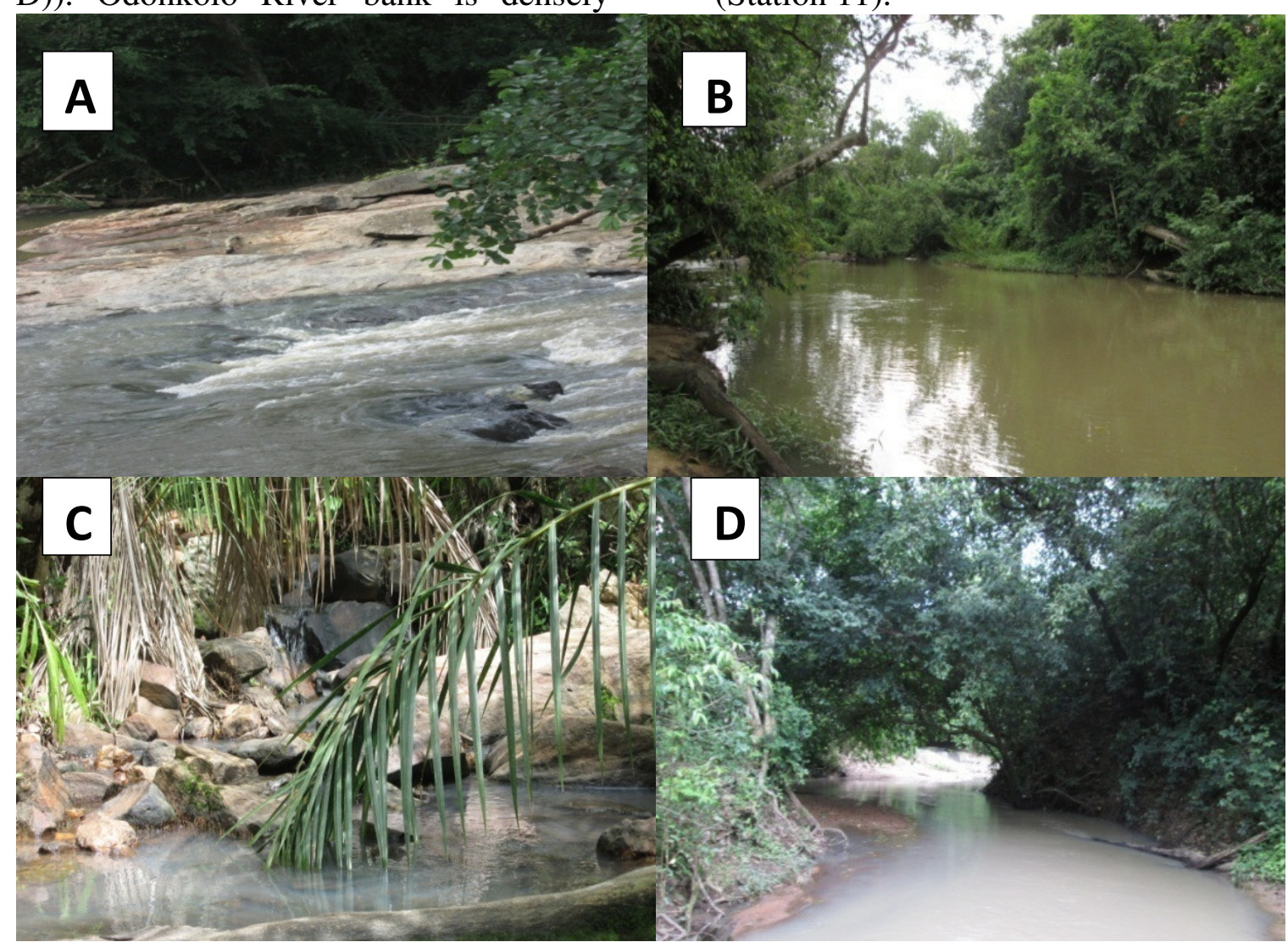

Plate 1: Some of the sampling sites at the location

\section{Collection of Samples}

\section{Phytobenthos Sampling}

Three replicate water samples were collected twice (March and June) to denotes the season (Dry and Wet). Due to the shallowness of the study site, water sampler was used directly to collect the water samples which were transferred into well labeled $500 \mathrm{ml}$ plastic containers with screw caps and preserved with $4 \%$ unbuffered formalin (Adesalu et al., 2015). Surface water samples for physico-chemical analysis were collected in 2litre container. Some analyses were done in-situ before taken the water samples for further analysis (Adesalu et 
al., 2015) Biological samples were analysed using relevant texts (Hustedt (1930-1937; 1954) Whitford and Schumacher (1973); Hendey (1964); Patrick and Reimer (1966, 1975), Krammer and Large-Bertalot (1986). Bukhtiyarova and Pomazkina, 2013.

\section{Macrobenthos Sampling}

As explained in Adesalu et al. (2016), at each station, soil samples were scooped directly at the sampling points due to shallowness of these sites. The sediments were sieved through a $0.5 \mathrm{~mm}$ mesh size stainless sieve. The sieve and its contents were immersed in the native water and gently agitated until organisms debris matrix removed and sieve contents transferred into properly labeled, wide mounted glass jars containing $4 \%$ unbufferred formalin solution for preservation. The macrobenthos was observed under the Olympus microscope at different magnifications and biological species documented. Identifications were made using appropriate keys (Needham and Needham, 1962; Quigley, 1977; Atobatele, et al., 2005; Ibemenuga and Inyang, 2006).

\section{Scanning Electron Microscopy}

Phytobenthos collections were prepared for light and scanning electron microscope observations. A portion of the cleaned sample was added to distilled water. This slurry was filtered onto a 0.45 $\mu \mathrm{m}$ Millipore type HA filter. The filters were allowed to dry. A portion of the filter was cut and mounted to an aluminum stub using adhesive carbon tape. The aluminum stub was sputter coated with $20 \mathrm{~nm}$ of gold or goldpalladium. All scanning electron microscope observations were performed with a JOEL JSM 6060LV using a $10 \mathrm{kV}$ accelerating voltage at St. Cloud State University, U.S.A.

\section{Physico-chemical Analysis}

Measurement of the surface water temperature was made in-situ with the aid of mercury-in-glass thermometer while water depth was measured using a pole and measuring tape. Surface water salinity was determined by using handheld refractometer; conductivity was determined by the use of Philips PW9505 Conductivity Meter (Range: 3$100,000 \mu \mathrm{S} / \mathrm{cm}$ and Automatic Temperature Compensation Unit) and the hydrogen ion concentration $(\mathrm{pH})$ was analyzed using the Cole Parmer Tester3 (an electronic $\mathrm{pH}$ meter). Total suspended solid and total dissolved solids were determined using the Gravimetric method (2540D APHA, 1998). Dissolved oxygen was determined using Titrimetric (Iodometric) method (Azide modification procedure (APHA, 1988).

\section{Community Structure Analysis}

To obtain the estimate of species diversity, three community structure indices were used: Margalef's diversity index (d), Shannon-Weaner Index $\left(\mathrm{H}^{1}\right)$ (Shannon and Weaner 1963) and Species Equitability (j) or Evenness (Pielou, 1975).

\section{Results}

\section{Physico-chemical Analysis}

Surface water samples recorded an average of $28^{\circ} \mathrm{C}$ and $32^{\circ} \mathrm{C}$ for both season (wet and dry) while the salinity $(0.03 \%$ $0.07 \%$ ) of the water showed a freshwater environment the $\mathrm{pH}$ ranged between 6.50 and 7.61 for both season Table 1 . 
Ethiopian Journal of Environmental Studies and Management Vol. 10 no.32017

Table 1: Physico-chemical parameters results obtained at the different stations (Average)

\begin{tabular}{|c|c|c|c|c|c|c|c|c|c|c|c|}
\hline & \multicolumn{11}{|c|}{ Stations } \\
\hline & & & Parts $\mathrm{C}$ & E Oinyi & iver & & & $\begin{array}{l}\text { Parts } \\
\text { Rive }\end{array}$ & f Odc & & $\begin{array}{l}\text { Odonkolo } \\
\text { River }\end{array}$ \\
\hline & 1 & 2 & 3 & 4 & 5 & 6 & 7 & 8 & 9 & 10 & 11 \\
\hline Air temperature $\left({ }^{\circ} \mathrm{c}\right)$ & 32 & 30.5 & 34.5 & 30 & 31 & 33 & 34.8 & 30.5 & 35 & 33 & 32.8 \\
\hline Water temperature $\left({ }^{\circ} \mathrm{c}\right)$ & 32 & 32 & 33 & 28.5 & 31.8 & 34 & 31 & 27.5 & 30.8 & 32 & 32.2 \\
\hline $\mathrm{pH}$ & 7.53 & 7.12 & 7.46 & 7.24 & 7.33 & 7.61 & 7.49 & 6.50 & 7.26 & 6.52 & 7.56 \\
\hline Salinity $(\% o)$ & 0.05 & 0.04 & 0.05 & 0.06 & 0.05 & 0.05 & 0.07 & 0.03 & 0.06 & 0.04 & 0.06 \\
\hline Depth (m) & 0.76 & 0.67 & 0.67 & 0.43 & 0.34 & 0.46 & 0.53 & 0.41 & 0.52 & 0.46 & 0.40 \\
\hline Conductivity $(\mu \mathrm{S} / \mathrm{cm})$ & 102.4 & 131.7 & 101.8 & 113.4 & 103 & 96.9 & 127.8 & 61.8 & 110 & 88.1 & 113 \\
\hline Total Dissolved Solids (mg/L) & 75.7 & 53.9 & 74.8 & 84.8 & 76.3 & 69.1 & 98.1 & 45.2 & 80.6 & 65.7 & 83.7 \\
\hline Dissolved Oxygen $(\mathrm{mg} / \mathrm{L})$ & 4.5 & 4.08 & 3.83 & 3.15 & 4.42 & 4.78 & 1.94 & 0.08 & 3.92 & 3.4 & 5.12 \\
\hline
\end{tabular}

\section{Phytobenthos}

The phytobenthos community of the Kaaba/Bunu locations of the sampling sites recorded 85 taxa distributed among five divisions Bacillariophyta, Chlorophyta, Charophyta, Euglenophyta and Cyanobacteria (Table 2). The composition of different groups among the eleven stations sampled is shown in Figure 1. Most species belong to the division, Bacillariophyta which is a diverse group with $78.28 \%$ of the identified species, followed by Euglenophytes $(7.43 \%)$ and Charophyta $(6.99 .0 \%)$ and The Chlorophytes and Cyanobacteria recorded $5.61 \%$ and $1.69 \%$ respectively, (Figure2). Among the diatoms, Pinnularia (10 species), Nitzschia (9 species), Navicula (7 species), Amphora (6 species) and Gomphonema (5 species) were the dominant genera. The Chlorophytes and Euglenophytes recorded 8 species each with six and three genera respectively. The blue green (Cyanobacteria) had three species with two genera, Chroococcus and Oscillatoria. Community structure analysis as shown in Figure 3 revealed the relationship between the Shannon Weaner index and Margalef (diversity) following almost same pattern. Scanning electron microscopy images of some species especially diatoms are presented on Plate 2. 
Table 2: Phytobenthos composition at the different locations in Kogi during dry and wet seasons at (cells/ml)

\begin{tabular}{|c|c|c|c|c|c|c|c|c|c|c|c|c|}
\hline & & \multicolumn{6}{|c|}{ Parts of Oinyi River } & \multicolumn{4}{|c|}{ Parts of Odogu River } & \multirow{2}{*}{$\begin{array}{c}\begin{array}{c}\text { Odonkolo } \\
\text { River }\end{array} \\
11\end{array}$} \\
\hline & & 1 & 2 & 3 & 4 & 5 & 6 & 7 & 8 & 9 & 10 & \\
\hline & & $\begin{array}{l}\left({\mathrm{N} 08^{\circ}}^{\circ} 00 .\right. \\
322^{\prime} \\
{\mathrm{E} 006^{\circ}}^{\circ} 17 . \\
\left.231^{\prime}\right)\end{array}$ & $\begin{array}{l}\left({\mathrm{N} 07^{\circ}}^{\circ} 58 .\right. \\
005^{\prime} \\
{\mathrm{E} 006^{\circ}}^{\circ} 15 . \\
\left.811^{\prime}\right)\end{array}$ & $\begin{array}{l}\left({\mathrm{N} 08^{\circ}}^{\circ} 00 .\right. \\
213^{\prime} \\
{\mathrm{E} 006^{\circ}}^{\circ} 16 . \\
\left.989^{\prime}\right)\end{array}$ & $\begin{array}{l}\left(\mathrm{N}^{\circ} 8^{\circ} 00 .\right. \\
253^{\prime} \\
{\mathrm{E} 006^{\circ}}^{\circ} 17 . \\
\left.419^{\prime}\right)\end{array}$ & $\begin{array}{l}\left(\mathrm{N}^{\circ} 8^{\circ} 00 .\right. \\
262^{\prime} \\
\mathrm{E}^{\prime} 06^{\circ} 16 . \\
\left.911^{\prime}\right)\end{array}$ & $\begin{array}{l}\left({\mathrm{N} 08^{\circ}}^{\circ} 00 .\right. \\
538^{\prime} \\
{\mathrm{E} 006^{\circ}}^{\circ} 16 . \\
\left.690^{\prime}\right)\end{array}$ & $\begin{array}{l}\left(\mathrm{N}^{\circ} 7^{\circ} 57 .\right. \\
296^{\prime} \\
\mathrm{E}^{\prime} 06^{\circ} 14 . \\
\left.419^{\prime}\right)\end{array}$ & $\begin{array}{l}\text { (N070 } 57 . \\
260^{\prime} \\
\mathrm{E} 006^{\circ} 14 . \\
\left.593^{\prime}\right)\end{array}$ & $\begin{array}{l}\left(\mathrm{N} 07^{\circ} 57 .\right. \\
454^{\prime} \\
\mathrm{E}^{\prime} 06^{\circ} 15 . \\
\left.250^{\prime}\right)\end{array}$ & $\begin{array}{l}\left(\mathrm{N}^{\circ} \mathrm{O} 56 .\right. \\
578^{\prime} \quad \text { E0066 } \\
\left.14.966^{\prime}\right)\end{array}$ & $\begin{array}{l}\left(\mathrm{N} 07^{\circ} 58 .\right. \\
956^{\prime} \\
{\mathrm{E} 006^{\circ}}^{\circ} 20 . \\
\left.769^{\prime}\right)\end{array}$ \\
\hline & Division: Bacillariophyta & & & & & & & & & & & \\
\hline & Class:Bacillariophyceae & & & & & & & & & & & \\
\hline & Order: Aulacoseirales & & & & & & & & & & & \\
\hline & Family: Aulacoseiraceae & & & & & & & & & & & \\
\hline \multirow[t]{3}{*}{1} & $\begin{array}{l}\text { Aulacoseira. granulata var. } \\
\text { angustissima (O.Müll.) Simonsen }\end{array}$ & & 30 & & & & & & - & & - & - \\
\hline & Order: Bacillariales & & & & & & & & & & & \\
\hline & Family Bacillariaceae & & & & & & & & & & & \\
\hline 2 & Nitzschia fasciculate (Grunow) Grunow & 40 & _- & - & 40 & _- & - & 10 & _- & _- & _- & _ \\
\hline 3 & N. flexoides Geitler & 30 & _- & 20 & _- & _- & 10 & _- & _- & _- & _- & 10 \\
\hline 4 & $\begin{array}{l}\text { Nitzschia filiformis var. ignorata } \\
\text { (Krasske) Cleve }\end{array}$ & 40 & & 10 & 20 & & _ & _ & _ & $\ldots$ & $=$ & $\ldots$ \\
\hline 5 & N. flexoides Geitler & - & 40 & - & & 20 & - & _- & - & _- & - & _- \\
\hline 6 & N. ignorata Krasske & & 10 & - & 30 & 10 & - & - & - & - & - & - \\
\hline 7 & N. linearis W. Smith & 10 & - & & - & $\ldots$ & & _- & _- & _- & - & \\
\hline 8 & N. palea (Kutz) W.Sm & 10 & - & 20 & - & - & 30 & - & - & - & - & 30 \\
\hline 9 & N. sigmoidea $($ Nitzsch) W.Smith & & - & 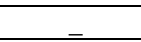 & _- & - & $\ldots$ & _- & _- & _- & - & 10 \\
\hline 10 & N. sublinearis Hustedt & 30 & - & - & - & - & - & - & - & - & - & _- \\
\hline \multirow[t]{3}{*}{11} & Nitzschia sp. & 10 & - & 10 & 40 & - & - & _- & - & _- & - & _- \\
\hline & Order: Cocconeidales & & & & & & & & & & & \\
\hline & Family: Cocconeidaceae & & & & & & & & & & & \\
\hline 12 & Cocconeis disculus (Schumann) Cleve & 20 & _- & _ & _- & _- & _ & _- & _- & _- & - & - \\
\hline \multirow{2}{*}{13} & Cocconeis placentulaEhrenberg & - & _- & 10 & _- & 10 & 20 & _- & 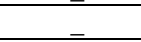 & _- & & 80 \\
\hline & Family: Achnanthidiaceae & & & & & & & & & & & \\
\hline 14 & Achnanthidium sp. & 60 & _- & _- & _- & _- & _- & _- & _- & _- & - & $\ldots$ \\
\hline \multirow[t]{3}{*}{15} & $\begin{array}{l}\text { Planothidium lanceolatum (Brébisson } \\
\text { ex Kützing) Lange-Bertalot }\end{array}$ & 10 & & - & $=$ & & & $=$ & $=$ & & & $=$ \\
\hline & Order: Coscinodiscales & & & & & & & & & & & \\
\hline & Family: Coscinodiscaceae & & & & & & & & & & & \\
\hline \multirow[t]{3}{*}{16} & Coscinodiscus sp. & _- & _- & _- & _- & _- & _- & _- & _- & 100 & _- & _- \\
\hline & Order: Cymbellales & & & & & & & & & & & \\
\hline & Family: Cymbellaceae & & & & & & & & & & & \\
\hline 17 & Cymbella silesiaca Bleisch & $\ldots$ & 10 & & _ & 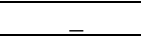 & & _ & _- & _ & $=$ & - \\
\hline \multirow[t]{2}{*}{18} & Cymbella sp. & - & - & 20 & - & 10 & 20 & - & - & - & 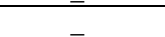 & 10 \\
\hline & Family: Gomphonemataceae & & & & & & & & & & & \\
\hline
\end{tabular}




\begin{tabular}{|c|c|c|c|c|c|c|c|c|c|c|c|c|}
\hline 19 & Gomphonema angustatum Agardh & & 10 & - & _- & & _- & _. & _- & _- & _. & _- \\
\hline 20 & G. clavatum Ehrenberg & 10 & 10 & - & - & 10 & _. & - & - & - & - & _. \\
\hline 21 & G. subclavatum (Grunow) Grunow & & 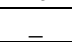 & $-\overline{10}$ & - & 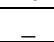 & $\overline{10}$ & - & - & - & - & - \\
\hline 22 & G. minutum C. Agardh) C. Agardh & 10 & - & - & - & - & - & - & - & - & - & - \\
\hline \multirow[t]{3}{*}{23} & G. parvulum (Kützing) Kützing & 10 & - & - & - & - & - & - & - & - & - & - \\
\hline & Order: Eunotiales & & & & & & & & & & & \\
\hline & Family: Eunotiaceae & & & & & & & & & & & \\
\hline \multirow[t]{3}{*}{24} & $\begin{array}{l}\text { Eunotia subarcuatoides Alles, Nörpel \& } \\
\text { Lange-Bertalot }\end{array}$ & _- & _- & 10 & _- & _- & _- & _ & _- & _- & 10 & _- \\
\hline & Order: Fragilariales & & & & & & & & & & & \\
\hline & Family: Fragilariaceae & & & & & & & & & & & \\
\hline 25 & Fragilaria sp. & 70 & 20 & _- & - & 10 & - & _. & - & _ & 10 & _- \\
\hline \multirow[t]{3}{*}{26} & Synedra sp. & 50 & 50 & - & 10 & & $\overline{70}$ & - & - & - & _- & 10 \\
\hline & Order: Licmophorales & & & & & & & & & & & \\
\hline & Family: Ulnariaceae & & & & & & & & & & & \\
\hline 27 & Ulnaria ulna (Nitzsch) Compère & - & - & 30 & 10 & 30 & 60 & _- & - & _ & _- & 10 \\
\hline 28 & U. ulna var. contracta (Østrup) Morales & 60 & - & - & - & - & - & - & - & - & - & - \\
\hline 29 & $\begin{array}{l}\text { Ulnaria danica (Kützing) Compère \& } \\
\text { Bukhtiyarova }\end{array}$ & _ & 10 & _ & 10 & - & & _- & _ & $=$ & _- & _- \\
\hline 30 & $\begin{array}{l}\text { U. ulna var. oxyrynchus (Kützing) } \\
\text { Aboal }\end{array}$ & 10 & 10 & & 30 & 10 & & _- & _- & _- & _- & 10 \\
\hline \multirow[t]{3}{*}{31} & Ulnaria sp. & - & 20 & $\overline{10}$ & - & 10 & $\overline{10}$ & - & - & - & - & 20 \\
\hline & Order: Naviculales & & & & & & & & & & & \\
\hline & Family: Amphipleuraceae & & & & & & & & & & - & \\
\hline 32 & Frustulia rhomboids (Ehr.) de Toni & _- & 10 & _- & _- & _- & _- & _- & _- & _ & _- & _- \\
\hline 33 & $\begin{array}{l}\text { F. rhomboids var. crassinervia } \\
\text { ((Brébisson ex W.Smith)) Ross }\end{array}$ & _- & 10 & _- & _- & _- & _- & _ & _ & _ & _ & _- \\
\hline \multirow[t]{2}{*}{34} & F. vulgaris (Thwaites) De Toni & - & 10 & - & - & & - & - & & - & - & \\
\hline & Family: Naviculaceae & & & & & & & & & $=$ & & \\
\hline 35 & $\begin{array}{l}\text { Gyrosigma scalproides (Rabenhorst) } \\
\text { Cleve }\end{array}$ & & 30 & & & & & & & & & 10 \\
\hline 36 & $\begin{array}{l}\text { Navigeia decussis (Østrup) } \\
\text { Bukhtiyarova }\end{array}$ & 20 & 10 & & & & & & & $\ldots$ & _- & \\
\hline 37 & Navicula exigua (Greg.) O. Muller & 30 & 70 & 30 & & 20 & 20 & & & $=$ & - & 30 \\
\hline 38 & N. mutica Kutzing & 20 & 180 & 110 & -- & 40 & 20 & - & - & - & - & 10 \\
\hline 39 & N. placenta Ehr. & & 10 & 30 & & 10 & & & & - & - & \\
\hline 40 & N. pupila Kutz. & 40 & 390 & 190 & 120 & 30 & 20 & 10 & 10 & - & - & $\overline{100}$ \\
\hline \multirow[t]{2}{*}{41} & N. radiosa (Kutz.) & 110 & _ & 50 & 10 & 10 & _- & - & 10 & - & - & 10 \\
\hline & Family: Neidiaceae & & & & & & & & & & & \\
\hline \multirow[t]{2}{*}{42} & Neidiumsp. & _- & _- & 10 & _- & _- & _- & _- & _- & _ & _- & - \\
\hline & Family: Pinnulariaceae & & & & & & & & & & & \\
\hline 43 & Pinnularia abaujensis (Plant.) Ross & _ & _. & _- & 10 & _- & _- & _- & _- & _ & $\ldots$ & - \\
\hline 44 & P. acrosphaeria Smith & - & - & - & $\ldots$ & 20 & - & 10 & - & - & - & - \\
\hline 45 & P. legumen Ehr. & - & - & - & - & _ & - & - & $\overline{10}$ & - & - & - \\
\hline 46 & $P$. lundii Hustedt & $\overline{70}$ & & & & & - & & 10 & - & - & \\
\hline
\end{tabular}




\begin{tabular}{|c|c|c|c|c|c|c|c|c|c|c|c|c|}
\hline 47 & P. macilenta Ehr. & 160 & 460 & 130 & 270 & 130 & 30 & 10 & _- & 10 & 30 & 110 \\
\hline 48 & P. maior (Kutzing) Rabenhorst & _ & - & 10 & 10 & - & _- & - & _- & _- & _- & _- \\
\hline 49 & P. microstauron (Ehrenberg) Cleve & 20 & - & & 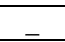 & & - & _- & - & _- & - & \\
\hline 50 & P. subcapitata Gregory & 10 & - & 30 & _- & 40 & - & _- & _- & _- & - & 10 \\
\hline 51 & P. viridis (Nitz.) Ehrenberg & & & 10 & _ & & - & _- & _- & _ & _ & \\
\hline \multirow[t]{2}{*}{52} & Pinnularia sp. & 30 & 40 & _- & _ & _- & - & _- & - & _- & _- & 20 \\
\hline & Family: Pleurosigmataceae & & & & & & & & & & & \\
\hline \multirow[t]{2}{*}{53} & Pleurosigma sp. & 90 & 80 & 40 & 40 & 20 & _- & _- & 10 & _- & _- & 10 \\
\hline & Family: Stauroneidaceae & & & & & & & & & & & \\
\hline 54 & $\begin{array}{l}\text { Stauroneis phoenicenteron (Nitzsch) } \\
\text { Ehrenberg }\end{array}$ & _- & 10 & 80 & _- & _- & _- & 10 & _- & _- & _- & _- \\
\hline \multirow[t]{3}{*}{55} & Stauroneis $\mathrm{sp}$. & _. & 20 & - & - & - & - & - & - & - & - & 10 \\
\hline & Order: Rhopalodiales & & & & & & & & & & & \\
\hline & Family: Rhopalodiaceae & & & & & & & & & 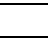 & & \\
\hline 56 & Rhopalodia gibba(Ehr.) Muller & 40 & 20 & 310 & 10 & 10 & 40 & _. & __ & __ & __ & 100 \\
\hline \multirow[t]{3}{*}{57} & Rhopalodia sp. & - & 40 & 170 & - & - & 20 & - & - & - & - & 130 \\
\hline & Order: Stephanodiscales & & & & & & & & & & & \\
\hline & Family: Stephanodisceae & & & & & & & & & & & \\
\hline \multirow[t]{3}{*}{58} & Cyclotella sp. & 10 & 20 & & & & _ & 10 & _- & _- & _- & \\
\hline & Order: Surirellales & & & & & & & & & & & \\
\hline & Family: Surirellaceae & & & & & & & & & & & \\
\hline 59 & Cymatopleura solea (Breb) W. Sm & 30 & 270 & 10 & 120 & 50 & _. & _. & _. & _ & _. & 10 \\
\hline \multirow[t]{3}{*}{60} & Surirella elegans Ehrenberg & 10 & - & - & - & 10 & _- & _- & _- & _ & _- & _. \\
\hline & Order: Thalassiophysales & & & & & & & & & & & \\
\hline & Family: Catenulaceae & & & & & & & & & & & \\
\hline 61 & $\begin{array}{l}\text { Amphora coffeaeformis (Agardh) } \\
\text { Kützing }\end{array}$ & _ & & 30 & _ & 10 & _ & _ & _ & _ & _ & 20 \\
\hline 62 & A. commutate Grunow & _- & 10 & & _- & 20 & _- & _- & _- & _- & _- & _- \\
\hline 63 & A. delicatissima Krasske & _- & _- & 10 & _- & _. & _- & _. & 10 & _- & __ & _. \\
\hline 64 & A. holsatica Hustedt & 10 & - & - & _ & - & _ & - & - & _ & _ & _- \\
\hline 65 & A. ovalis (Kutzing) Kutzing & 10 & - & 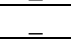 & & & - & _- & _- & _- & _- & \\
\hline \multirow[t]{5}{*}{66} & Amphora sp. & 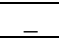 & 30 & 10 & _ & 10 & 20 & _ & _- & _ & _ & 20 \\
\hline & Division: Charophyta & & & & & & & & & & & \\
\hline & Class: Zygnematophyceae & & & & & & & & & & & \\
\hline & Order: Desmidiales & & & & & & & & & & & \\
\hline & Family: Closteriaceae & & & & & & & & & & & \\
\hline 67 & $\begin{array}{l}\text { Closterium acerosum Ehrenberg ex } \\
\text { Ralfs }\end{array}$ & & & & & & & & & & & 20 \\
\hline \multirow[t]{2}{*}{68} & $\begin{array}{l}\text { C. closterioides (Ralfs) A. Louis \& } \\
\text { Peters }\end{array}$ & $=$ & $=$ & & 10 & & $=$ & _- & _- & 10 & _- & \\
\hline & Family: Desmidiaceae & & & & & & & & & & & \\
\hline \multirow[t]{2}{*}{69} & Cosmarium sp. & _- & _- & 20 & _- & 20 & _ & _- & _ & _ & _. & 10 \\
\hline & Family: Gonatozygaceae & & & & & & & & & & & \\
\hline \multirow[t]{2}{*}{70} & Gonatozygon sp. & _ & _ & 20 & 20 & _- & _. & _. & _- & _. & _ & 30 \\
\hline & Order: Zygnematales & & & & & & & & & & & \\
\hline
\end{tabular}




\begin{tabular}{|c|c|c|c|c|c|c|c|c|c|c|c|c|}
\hline & Family: Zygnemataceae & & & & & & & & & & & \\
\hline \multirow[t]{5}{*}{71} & Spirogyra sp. & _- & _- & _- & _ & _- & 500 & _ & _ & _ & _- & _- \\
\hline & Division: Chlorophyta & & & & & & & & & & & \\
\hline & Class: Chlorophyceae & & & & & & & & & & & \\
\hline & Order: Sphaeropleales & & & & & & & & & & & \\
\hline & Family: Scenedesmaceae & & & & & & & & & & & \\
\hline 72 & $\begin{array}{l}\text { Desmodesmus quadricauda (Turp.) } \\
\text { Breb. }\end{array}$ & 10 & & _- & _- & - & _- & 10 & _- & $=$ & _- & _- \\
\hline \multirow{2}{*}{73} & D. quaricaudavar. westiiG.M. Smith & _- & 10 & _- & _- & _- & _- & - & _- & & 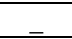 & 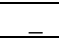 \\
\hline & Family: Microsporaceae & & & & & & & & & & & \\
\hline \multirow[t]{5}{*}{74} & Microspora sp. & _- & _- & _- & _- & _- & _- & 500 & _- & _- & _- & _- \\
\hline & Division: Euglenophyta & & & & & & & & & & & \\
\hline & Class: Euglenophyceae & & & & & & & & & & & \\
\hline & Order: Euglenales & & & & & & & & & & & \\
\hline & Family: Euglenaceae & & & & & & & & & & & \\
\hline 75 & Euglena acus var. rigida Huebner & _- & - & _ & _ & _ & _ & _ & - & 10 & - & - \\
\hline 76 & E. intermedia (Klebs) Schmitz & _ & & _ & _- & _ & _- & _ & _- & 10 & _- & _- \\
\hline 77 & E. proxima Dangeard & _- & 10 & _- & _- & _- & 7 & _- & 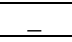 & & _ & - \\
\hline 78 & Euglena sp. & & 10 & _- & & 20 & 20 & & 20 & 430 & 10 & 10 \\
\hline \multirow[t]{2}{*}{82} & Trachelomonas sp. & 10 & - & _- & _- & - & - & _- & - & - & _- & _- \\
\hline & Family: Phacaceae & & & & & & & & & & & \\
\hline 79 & Phacus curvicauda Svirenko & $\ldots$ & _- & _ & _- & $\ldots$ & _ & 10 & _- & 10 & __ & _. \\
\hline 80 & P. longicauda (Ehrenb.) Dujardin & - & - & _- & & & _- & 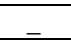 & _- & 30 & _- & _ \\
\hline \multirow[t]{5}{*}{81} & Phacus sp. & _- & _- & _ & 10 & 40 & _ & 1 & _ & 40 & _ & - \\
\hline & Division: Cyanobacteria & & & & & & & & & & & \\
\hline & Class: Cyanophyceae & & & & & & & & & & & \\
\hline & Order: Chroococcales & & & & & & & & & & & \\
\hline & Family: Chroococcaceae & & & & & & & & & & & \\
\hline \multirow[t]{3}{*}{83} & Chroococcus sp. & _ & 10 & _- & _ & _ & _ & _ & _ & _ & _- & _- \\
\hline & Order: Oscillatoriales & & & & & & & & & & & \\
\hline & Family: Oscillatoriaceae & & & & & & & & & & & \\
\hline 84 & Oscillatoria agardii Gomont & 60 & & & & & & & & & & \\
\hline \multirow[t]{6}{*}{85} & Oscillatoria sp. & & - & & 10 & - & - & 80 & _- & _- & _- & - \\
\hline & Total number of species & 37 & 35 & 30 & 21 & 27 & 17 & 11 & 6 & 9 & 4 & 28 \\
\hline & Total number of individuals & 1270 & 1980 & 1450 & 890 & 630 & 920 & 661 & 70 & 650 & 60 & 860 \\
\hline & Margalef (d) & 5.04 & 4.48 & 3.98 & 2.94 & 4.03 & 2.34 & 1.54 & 1.18 & 1.24 & 0.73 & 4.00 \\
\hline & Shannon-Weiner (H1) & 3.25 & 2.58 & 2.74 & 2.36 & 2.96 & 2.60 & 1.41 & 1.75 & 1.20 & 1.24 & 2.84 \\
\hline & Species evenness $(\mathrm{j})$ & 0.90 & 0.73 & 0.09 & 0.78 & 0.90 & 0.92 & 0.59 & 0.98 & 0.55 & 0.89 & 0.85 \\
\hline
\end{tabular}




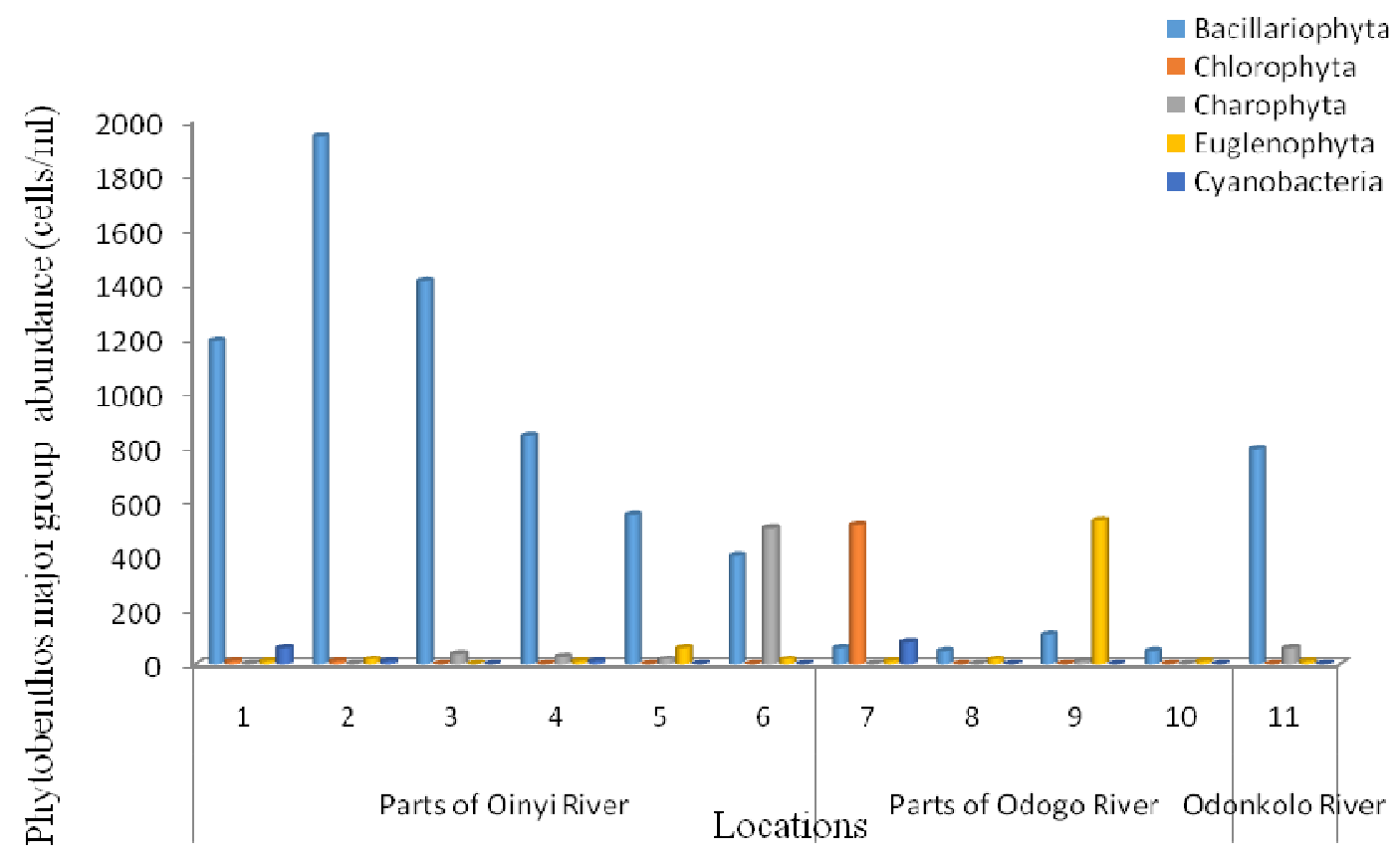

Figure 1: Comparison of different phytobenthos group abundance at different locations in Kaaba/Bunu Kogi

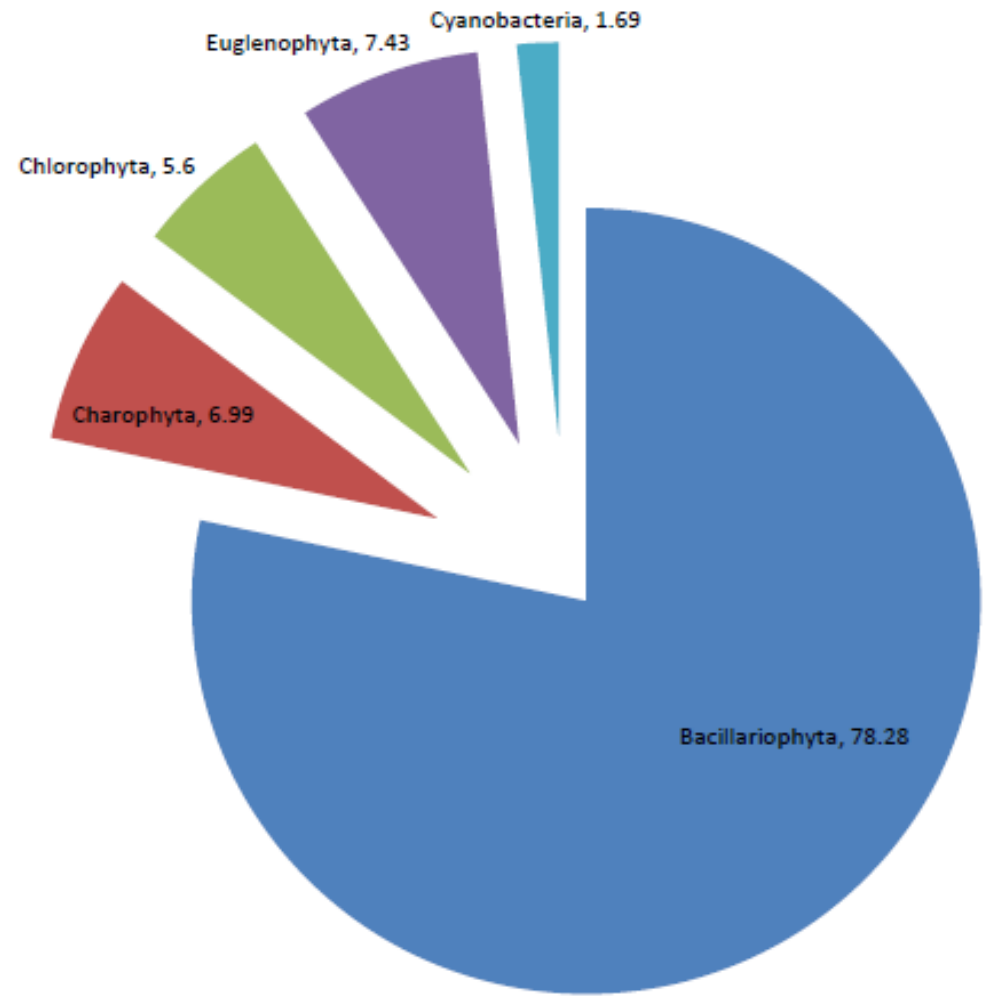

Figure 2: Percentage composition of major groups of phytobenthos 


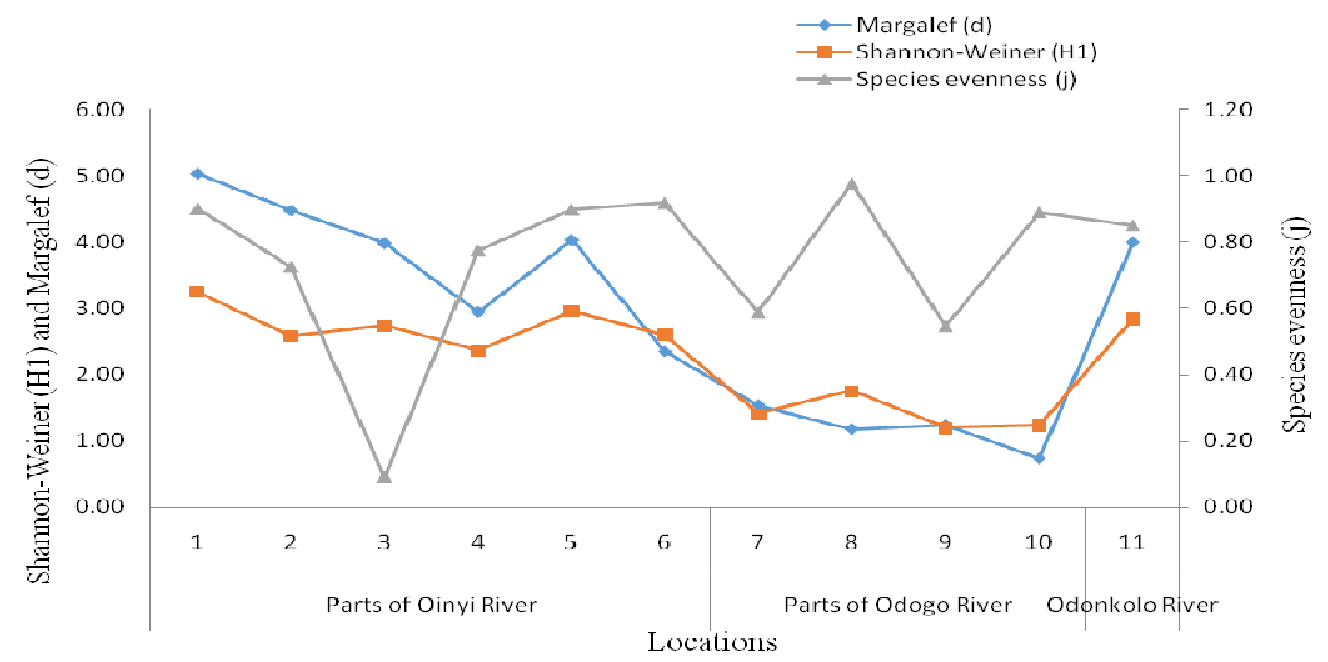

Figure 3: Community structure analysis at Kaaba/Bunu, Kogi locations
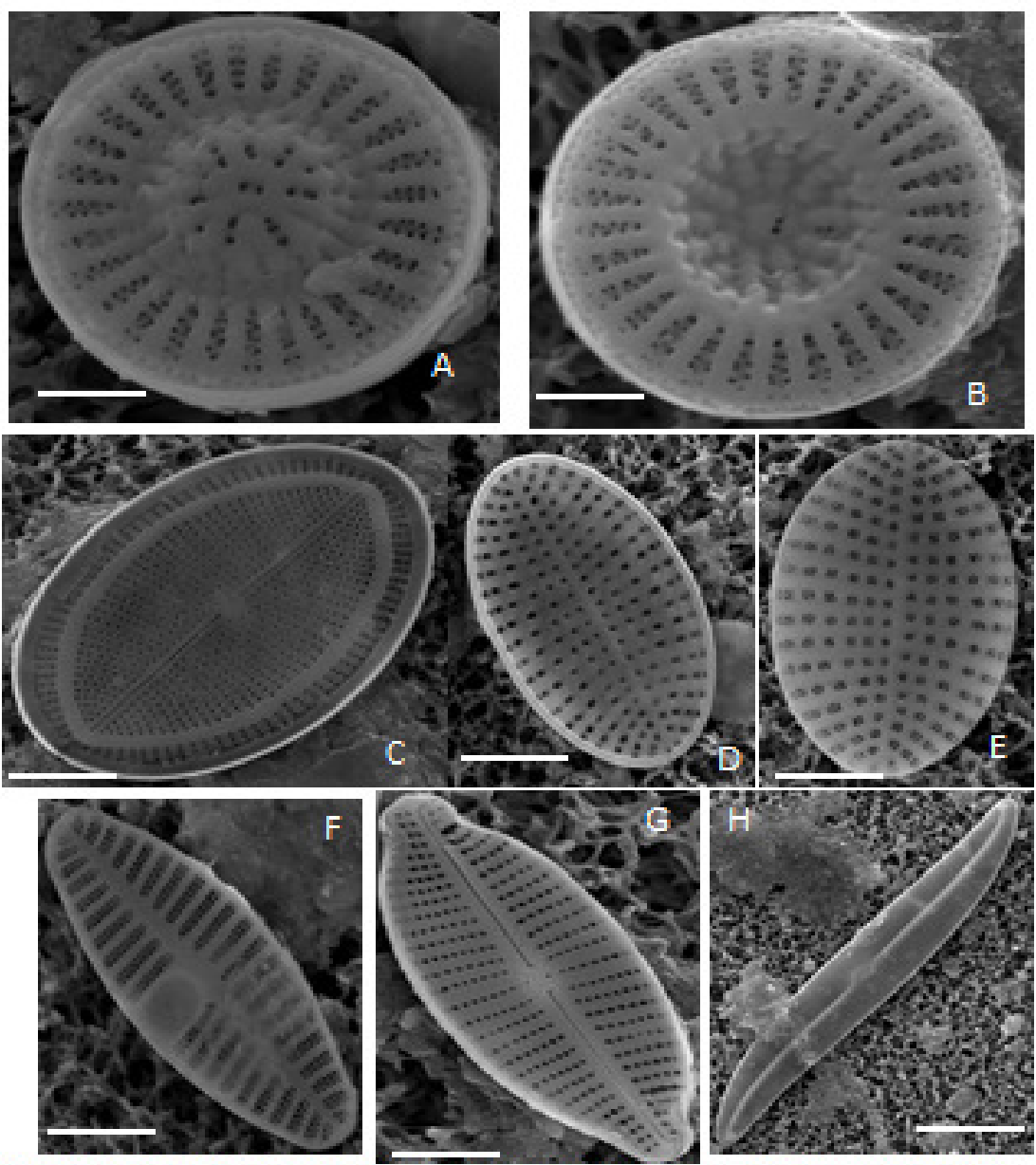

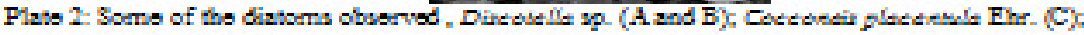

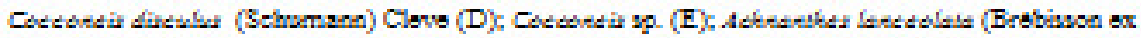

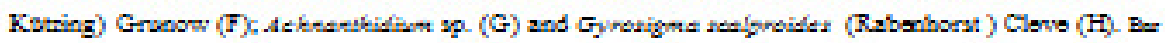
$-10 \mathrm{n}$ 


\section{Macrobenthos}

The analysis for the benthic samples was presented on Table 3 .The macro benthic invertebrates were represented by 14 taxa, belonging to 3 groups (Table $3)$.The dominant groups were the Gastropoda and Oligochaeta with each accounting for $35.71 \%$ while the Insecta group accounted for $28.57 \%$ of the total individuals respectively (Figures 4 and
5). The dominant taxa were Potamopyrgus and Lumbriculus species accounting for $35.71 \%$ each of the total individuals recorded while Aeschna and Chironomus species were least represented with $14.29 \%$ each of the total individuals recorded (Figure 6).The Shannon-Wiener Index (Hs), Margalef Index (d) and Equitability Index (j) were highest at some stations (Table 3 ).

Table 3.The distribution, occurrence and diversity indices of macro-benthic invertebrate community at the study stations

\begin{tabular}{|c|c|c|c|c|c|c|c|c|c|c|c|}
\hline & \multicolumn{11}{|c|}{ Stations } \\
\hline & \multicolumn{6}{|c|}{ Parts of Oinyi River } & \multicolumn{4}{|c|}{ Parts of Odogo River } & \multirow{2}{*}{$\begin{array}{l}\text { Odonkolo } \\
\text { River }\end{array}$} \\
\hline & 1 & 2 & 3 & 4 & 5 & 6 & 7 & 8 & 9 & 10 & \\
\hline \multicolumn{12}{|l|}{ GASTROPODA } \\
\hline Potamopyrgus spp. & 1 & - & & & 1 & 3 & - & & - & - & - \\
\hline \multicolumn{12}{|l|}{ INSECTA } \\
\hline Aeschna spp. & - & - & - & - & & 1 & - & 1 & - & & \\
\hline Chironomus spp. & - & - & - & - & 1 & & - & & - & & 1 \\
\hline \multicolumn{12}{|l|}{ OLIGOCHAETA } \\
\hline Lumbriculus spp. & 1 & - & - & - & 1 & 1 & - & & - & 1 & 1 \\
\hline Total species diversity (S) & 2 & - & - & - & 3 & 3 & - & 1 & - & 1 & 2 \\
\hline Total abundance $(\mathrm{N})$ & 2 & - & - & - & 3 & 5 & - & 1 & - & 1 & 2 \\
\hline Log of Species diversity $(\log S)$ & 0.30 & - & - & - & 0.45 & 0.45 & - & 0.00 & - & 0.00 & 0.30 \\
\hline Log of abundance $(\log N)$ & 0.30 & - & - & - & 0.45 & 0.7 & - & 0.00 & - & 0.00 & 0.30 \\
\hline Shannon-Wiener Index (Hs) & 0.30 & - & - & - & 0.48 & 0.41 & - & 0.00 & - & 0.00 & 0.30 \\
\hline Margalef Index (d) & 1.45 & - & - & - & 1.82 & 1.24 & - & 0.00 & - & 0.00 & 1.45 \\
\hline Equitability Index (j) & 1.00 & - & - & - & 1.07 & 0.91 & - & 0.00 & - & 0.00 & 1.00 \\
\hline
\end{tabular}


- OLIGOCHAETA $=$ GASTROPODA $\square$ INSECTA

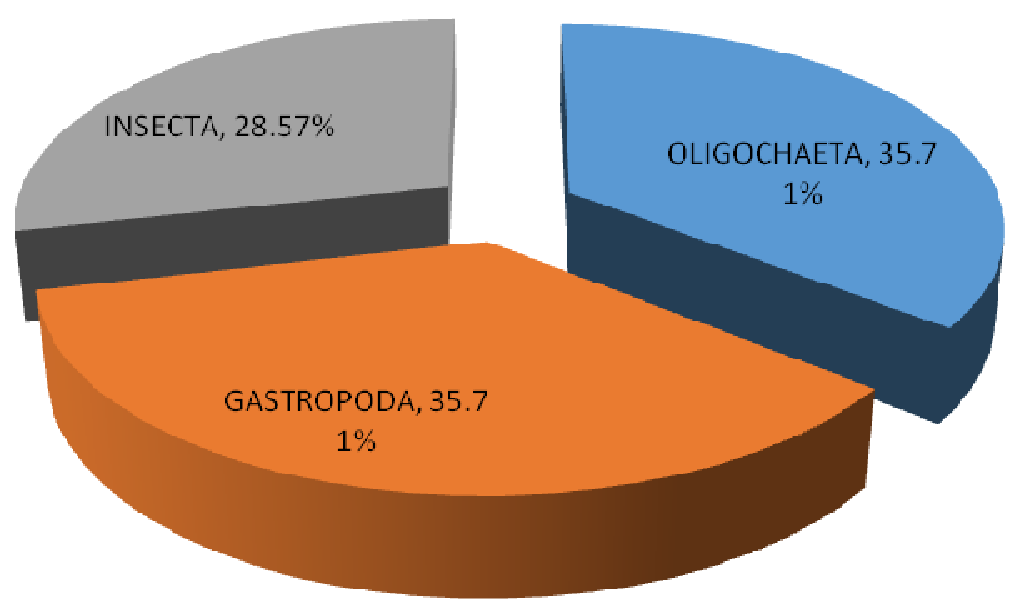

Figure 4: Percentage contribution of the major macro-benthic invertebrate groups at the study stations

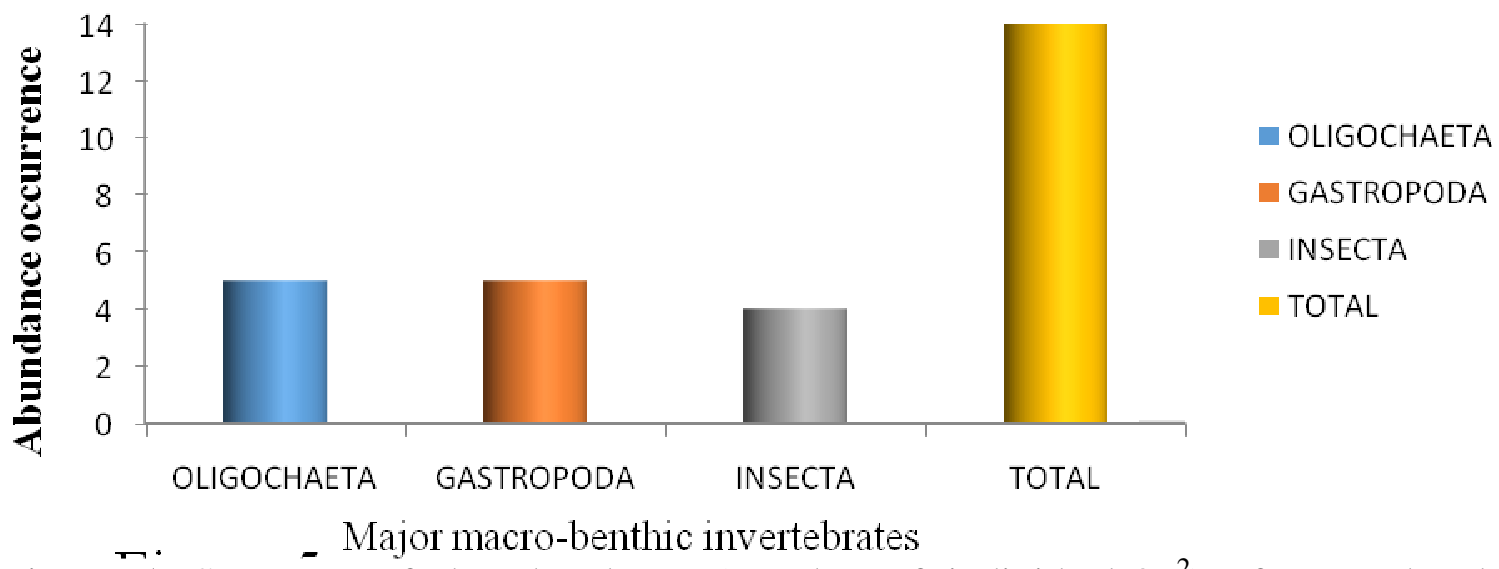

Figure 5: Summary of the abundance (Number of individuals $/ \mathrm{m}^{2}$ ) of macro-benthic invertebrate groups at the study stations

\section{Discussion}

The relative abundance of diatoms in these rivers may be as a result of the shallowness which paved way for deep light penetration which supports the rate of photosynthesis. The dominance of diatoms over other group confirms earlier reports made by Chindah and Pudo (1991) in Bonny River; Erondu and Chindah (1991) in the newCalabar River, Niger Delta; Adesalu (2008) in Lekki lagoon; Adesalu and Nwankwo (2005, 2008) in Olero and Abule Eledu creek respectively; Adesalu and Kunrunmi (2012) in the Lagoons of South-Western Nigeria, Adesalu and Kunrunmi (2016) for Majidun creek; Adesalu et al. (2008, 2014, 2015) in Ogbe and Ipa-Itako creeks and Majidun. However, the low number in the population of macro-benthic invertebrates recorded at the study stations could be due to the 
developmental rate of small macroinvertebrates, since most aquatic invertebrates are benthic only at larval stages while their adult lives are spent outside aquatic environments (Ibemenuga and Inyang, 2006). The total number of 14 taxa reported in this present study is far less than those reported for rivers elsewhere (Edema et al., 2002; Adakole and Anunne, 2003, Adesaluet al., 2016a and $b$ ) and these may be as a result of different environmental conditions such as water quality and movement, substrate instability and food availability (Esenowo and Ugwumba (2010). The water body as described previously, especially the Oinyi river is seasonal and this was observed in dry season as most of the river course was dry while in wet season, the water was not evenly distributed. Parts of the river were also dried while some parts were flooded in the wet season but no fish was caught.

\section{Conclusion}

The dominant of diatoms species in this study supported other reports from similar water bodies in Nigeria while the absence of Eunotia species conformed to the fact that they thrive very well in acidic water in this case the water is essentially neutral. However, the euglenoids, an indicator of organically polluted area which was observed in some of the stations especially Station 10 is probably due to the closeness of this station to settlements where domestic wastes get into the water body unabated. The paucity of benthic fauna in these rivers might be due to the nature of the river bed.The observation of desmids, an indicator of nutrient poor water (oligotrophic) also implies that the rivers still support life as depicted by high dissolved oxygen value..

\section{Acknowledgements}

Authors are most grateful to anonymous environmental firm for providing the logistics.

\section{References}

Adeoye, N.O. (2012). Spatio-Temporal Analysis of Land Use/Cover Change of Lokoja - A Confluence Town. Journal of Geography and Geology; 4(4):40-51. DOI: http://dx.doi.org/10.5539/jgg.v4n4p 40

Adesalu, T.A. (2008). Phytoplankton dynamics in relation to water quality indices in Lekki lagoon. Ph.D thesis, University of Lagos.

Adesalu, T.A. and Nwankwo, D.I. (2005). Studies on the phytoplankton of Olero creek and parts of Benin River, Nigeria.The Ekologia, 3(2):21-30.

Adesalu, T.A. and Nwankwo, D.I. (2008). Effect of water quality indices on phytoplankton of a sluggish tidal creek in Lagos, Nigeria. Pakistan Journal of Biological Sciences, $\quad$ 11: $\quad 836-$ 844.DOI: 10.3923/pjbs.2008.836.844

Adesalu, T.A., Abiola, T.O. and Bofia, O.T. (2008). Studies on the epiphytic algae associated with two floating aquatic macrophytes in a sluggish non-tidal polluted creek in Lagos, Nigeria. Asian Journal of Scientific Research 1: 363-373.

DOI:10.3923/ajsr.2008.363.373

Adesalu, T.A., Adesanya, T. and Ugwuzor, C. (2014). Phytoplankton composition and water chemistry 
of a tidal creek (Ipa -Itako) part of Lagos Lagoon. Journal of Ecology and Natural Environment, 6 (11): $\quad 373-388 . \quad$ DOI: 10.5897/JENE2014. 0473

Adesalu, T. A, Kunrunmi, O.A. and Lawal, M.O. (2015). Diversity of plankton and macrobenthos of freshwater habitats in Kogi State, Nigeria. Centrepoint Journal (Science Edition), 21(1):35- 53.

Adesalu, T.A and Kunrunmi, O.A. (2016). Diatom communities in riparian systems associated with Lagos lagoon, Nigeria 1. Seasonal and anthropogenic patterns in Majidun Creek. Algological Studies, 150: 39-52.DOI: 10.1127/algol_stud/2016/0249

Adesalu, T.A., Kunrunmi, O.A. and Lawal, M.O. (2016a). Plankton and macrobiota communities of three tropical freshwater habitats in Ogun and Ondo States, South-west,

Nigeria. Notulae Scientia Biologicae, 8(2): 246-255. DOI: 10.15835/nsb.8.2.9792

Adesalu, T.A., Kunrunmi, O.A. and Lawal, M.O. (2016b). Water quality Assessment: A case study of plankton and macrobenthic invertebrates of Porto-Novo and parts of Gulf of Guinea, Journal of Aquatic Sciences,31(1A):39-66. http://dx.doi.org/10.4314/jas.v31i1. $\underline{4}$

Atobatele, O.E., Morenikeji, O.A. and Ugwumba, O.A. (2005). Spatial Variation in Physical and Chemical Parameters and Benthic Macroinvertebrate Fauna of River Ogunpa, Ibadan. The Zoologist 3: 58-67.
Adakole, J.A. and Anunne, P.A. (2003). Benthic macroinvertebrates as indicators of environmental quality of an urban stream in Zaria, Northern Nigeria. J. Aqua. Sci., 18:85-92. http://dx.doi.org/10.4314/jas.v18i2. 19948

Biggs, B.J.F., Stevenson, .R.J. and Lowe, R.L. (1998). A habitat matrix conceptual model for stream periphyton. Arch Hydrobiol., 143:21-56.

Bukhtiyarova, L.N. and Pomazkina, G.V. (2013). Bacillariophyta of Lake Baikal.Volume 1. Genera Baikalia, Slavia, Navigeia, Placogeia, Grachevia, Goldfishia, Nadiya, Cymbelgeia. pp. [1]-184, 278 figs in 110 pls. Lviv: Lega-Pres.

Chindah, A.C. and Pudo, J. (1991). A preliminary checklist of algae found in plankton of bonny River in Niger Delta, Nigeria. Fragm. Flor. Geobat. 36: 112-125.

Edema, C.U., Ayeni, J.O and Aruoture, A. 2002.Some observations on the zooplankton and macrobenthos of the Okhuo River, Nigeria. Journal Aquatic Science 17: 145-149. http://hdl.handle.net/123456789/77 9

Erondu, E.S. and Chindah, A.C. (1991). Physico-chemical and phytoplankton changes in a tidal freshwater station of the New Calabar River South Eastern Nigeria. Environmental Ecology, 9:561-570.

Esenowo, I.K and Ugwumba, A.A.A. (2010). Composition and abundance of macrobenthos in Majidun River, Ikorodu, Lagos 
State, Nigeria. Research Journal of Biological Sciences, 5(8): 556560.DOI:

10.3923/rjbsci.2010.556.560

Field, C.B., Behrenfeld, M.J., Randerson, J.T. and Falkowski, P.G. (1998). Primary production of the biosphere: Integrating terrestrial and oceanic components. Science 281, $237240 . \quad$ DOI: 10.1126/science.281.5374.237

George, A.D.I., Abowei, J.F.N. and Daka, E.R. (2009). Benthic macroinvertebrate fauna and physico-chemical parameters in Okpoka creek sediments, Niger Delta, Nigeria. International Journal of Animal and Veterinary Advances, 1: 59-65. ISSN: 20412908

Hendey, N.I. (1964). An introductory account of algae of Bristish Coastal waters. Part V. Bacillariophyceae (diatoms) London, Fisheries investigations Series 4, 1-317pp.

Hustedt, F. (1930-1937). Die. Kiselalgen 7. In Rabenhorst (Ed.) Kryptogemen-flora von DeutshlandÖsterreichsun derSchweiz. A Kademiscehe VergasellSchaft M.L.H. Leipzing. 466p.

Hustedt, F. (1954). Die Diatomeenflora der Eifelmaare. Archiv für Hydrobiologie 48(4): 451- 496.

Ibemenuga, K.N. and Inyang, N. (2006). Macroinvertebrate Fauna of a Tropical Freshwater Stream in Nigeria. Animal Research International 3(3):553561.http://dx.doi.org/10.4314/ari.v3 i3.40791

Krammer, $\mathrm{H}$. and Large-Bertalot, $\mathrm{H}$. (1986). Bacillariophyceae. 1. Teil:
Naviculaceae In: Ettl, H., Gerloff, J., Heynig, H., Mollenhauer, D., (Hrsgb.), Süsswasserflora von Mitteleuropa. Bd. 2 Fischer Verlag, Stuttgart. 876pp.

Marinković, N., Krizmanić, J., Karadžić, V., Karadžić,1.B., Vasiljević, B. and Paunović, M. (2016). Algal diversity along the Serbian stretch of the Sava River. Water Research and Management,6(2):2733.http://www.wrmjournal.com/ind ex.php?option=com_content\&view $=$ article $\& i d=352 \&$ Itemid $=292$

Mulholland, P.J. (1996). Role in nutrient cycling in streams. In: Stevenson R.J., Bothwell, M.L, Lowe R.L (eds) Algal ecology: freshwater benthic ecosystems. Academic, San Diego, 605-639pp.

Needham, J.G. and Needham, P.R. (1962). A guide to the study of freshwater biology. Holden-Day Inc. San Francisco. 108pp.ISBN13:978-0070461376

Patrick, R. and Reimer. C.W. (1966). The diatoms of the United States exclusive of Alaska and Hawaii. Vol. 1.Fragilariaceae, Eunotiaceae, Achnanthaceae, Naviculaceae. Monographs of The Academy of Natural Sciences of Philadelphia. No. 13.688 pp.

Patrick, R. M. and Reimer, C. W. (1975). The diatoms of the United States, exclusive of Alaska and Hawaii. Volume 2, Part 1 Academy of Natural Sciences of Philadelphia, Pennsylvania .213 pp.

Pielou, E.C. (1975). Ecological diversity, New York, John Willey and Sons, 165pp. ISBN: 0471689254

Quigley, M. (1977). Invertebrates of streams and rivers: A key to 
identification. Edward Arnold (Publishers) Ltd, London. 81pp.ISBN-10: 0713100915

Shannon, C.E. and Weaver, W. (1963). The mathematical theory of communication. University of Illinois, Press, Urbana, Illinois, $125 \mathrm{pp}$.

Water framework Directive (2000). Water framework directiveDirective of European Parliament and of the council 2000/60/ECEstablishing a framework for community Action in the Field of Water Policy. European Union, the European Parliament and Council, Luxemburg.

Whitford, L.A. and Schumacher, G.J. (1973). A manual of fresh water algae. Sparks, North Carolina, USA, 324pp.ISBN-13:978- 